\title{
ERGODIC UNDEFINABILITY IN SET THEORY AND RECURSION THEORY
}

\author{
DANIELE MUNDICI
}

\begin{abstract}
Let $T$ be a measure preserving ergodic transformation of a compact Abelian group $G$ with normalized Haar measure $m$ on the collection $\mathscr{B}$ of Borel sets; call $g \in G$ generic w.r.t. a set $B \in \mathscr{B}$ iff, upon action by $T, g$ is to stay in $B$ with limit frequency equal to $m(B)$. We study the definability of generic elements in Zermelo-Fraenkel set theory with Global Choice (ZFGC, which is a very good conservative extension of ZFC), and in higher recursion theory. We prove (1) the set of those $g \in G$ which are generic w.r.t. all ZFGC-definable Borel subsets of $G$ is not ZFGC-definable, and (2) "being generic w.r.t. all hyperarithmetical properties of dyadic sequences" is not itself a hyperarithmetical property of dyadic sequences.
\end{abstract}

0. Introduction. We apply some well known facts of ergodic theory to obtain undefinability results in such languages as Zermelo-Fraenkel set theory with Global Choice, or the effective Borel Hierarchy of subsets of the circle.

Let $G$ be an infinite compact Abelian group with the collection $\mathscr{B}$ of Borel subsets of $G$, and $m$ the (unique) normalized Haar measure on $\mathscr{B}$ (see [Ha] and [Po]). Given any $B \in \mathscr{B}$, the probability $m(B)$ may be also evaluated by means of "generic" elements of $G$ as follows: let $T: G \rightarrow G$ be a measure preserving ergodic transformation of $(G, \mathscr{B}, m)$; such $T$ exists whatever $(G, \mathscr{B}, m)$ is (see [Wa] and [DGS]). Given now $g \in G$ and letting $\chi_{B}$ be the indicator of $B$, the limit

$$
\lim _{n \rightarrow \infty} n^{-1} \sum_{i=0}^{n-1} \chi_{B}\left(T^{i}(g)\right)
$$

almost surely (exists and) equals $m(B)$, by Birkhoff's theorem (see [Fr]); generalizing a definition of [DGS], call $g \in G$ generic w.r.t. $B$ (for measure $m$ ) iff the above limit exists and equals $m(B)$; intuitively, $g$ is generic w.r.t. $B$ iff $\left\{T^{i}(g)\right\}_{i \in \omega}$ provides a random sample of $B$, iff upon action by $T, g$ is to stay in $B$ with limit frequency equal to $m(B)$. If now $\mathcal{L} \subseteq \mathscr{B}$ is a countable field of subsets of $G$, let $G(\mathfrak{L})=\{g \in G \mid \forall B \in \mathcal{L} g$ is generic w.r.t. $B\}$. When $g \in G(\mathcal{L})$ we say that $g$ is generic w.r.t. $\mathcal{L}$ : The dependence of $G(\mathscr{L})$ also on $T$ and the actual topology of $G$ should be understood.

Now think of $\mathcal{E}$ as the collection of properties in (i.e. subsets of) $G$ which are definable in some language; the question arises: Is the property of being generic w.r.t. $\mathcal{L}$ definable in $\mathcal{L}$ ? We shall show (Theorem 1.1) that this is not the case provided $\mathcal{L}$ satisfies the following closure conditions: (i) each $B \in \mathcal{L}$ of measure one contains a singleton of $\mathcal{L}$, and (ii) $\mathcal{E}$ is closed under $T$.

Received by the editors November 5, 1974 and, in revised form, April 28, 1980.

AMS (MOS) subject classifications (1970). Primary 22D40, 03E15, 03E25, 04A15, 04A25, 03D55; Secondary 28D05, 54H05, 22C05, $03 \mathrm{C40}$. 
Our first application of Theorem 1.1 is to Zermelo-Fraenkel set theory with Global Choice (ZFGC), see [Le]. ZFGC has a new (unary function) symbol $s$ called selector, for global choice, and new axioms

(a) $x \neq \varnothing \rightarrow s(x) \in x$, and

(b) all instances of the axiom scheme of replacement with any formula of the language of $\mathrm{ZFGC}$.

ZFGC is a conservative extension of ZFC (i.e. Zermelo-Fraenkel set theory with Choice), in the sense that it adds no new theorems in the language of ZFC (see [Fe] and [Ga]); Thus, in particular, if ZFC is consistent (if $\mathrm{ZF}$ is consistent) then so is ZFGC, and ZFGC does not commit us to believe anything new about sets. Further, the presence of global choice gives ZFGC a nice property explained in the following discussion: As usual (see [Le, p. 12]) we call $\tau$ a class term iff $\tau$ is an expression of the form $\{x \mid \varphi(x)\}$, with $\varphi(x)$ an arbitrary formula of ZFGC; following [Le, p. 171 and p. 178] we call $C$ a definable class of ZFGC iff $C$ is given by a class term of ZFGC with no free variables; examples of definable classes are: $V$ (i.e. $\{x \mid x=x\}$ ), $\varnothing$ (i.e. $\{x \mid x \neq x\})$ and $S$ (i.e. $\{x \mid \exists y, x=s(y)\}$ ); the latter class cannot be proved in ZFGC to be equal to any class of ZF. We say that $r$ is a definable set of ZFGC (or, $r$ is ZFGC-definable) iff $r$ is a definable class which is also a set. One now sees immediately that any definable nonvoid set of ZFGC contains a definable singleton (proof: if $r=\{x \mid \varphi(x)\}$ then $\{y \mid \exists z(y=s(z) \wedge \forall x(x$ $\in z \leftrightarrow \varphi(x)))\}$ is a ZFGC-definable singleton contained in $r$ ).

The above nice property, together with conservativity mentioned before makes ZFGC a very good extension of ZFC.

Returning to $(G, \mathscr{B}, m)$, assume that $T: G \rightarrow G$ is measure preserving, ergodic and, in addition, satisfies the following condition: $\forall g \in G$ if $\{g\}$ is ZFGC-definable then so is

$$
\bigcup_{i \in \omega}\left\{T^{i}(g)\right\}
$$

Then, in Corollary 1.2, we prove that the set of all those $g \in G$ which are generic w.r.t. all Borel ZFGC-definable subsets of $G$ is a Borel subset of $G$ which is not ZFGC-definable.

Our second application is to higher recursion theory: Let $G=K \subseteq \mathbf{C}$ be the unit circle with complex multiplication, natural topology, normalized Haar measure $m$ on the Borel sets; let $T: K \rightarrow K$ be given by $T(z)=z^{2}$ for any $z \in K$; let $\lambda$ : $K \rightarrow[0,1)$ isomorphically embed $K$ onto $[0,1)$, where $\lambda=\varphi^{-1}$ and

$$
\varphi(\theta)=e^{2 \pi i \theta}, \quad \forall \theta \in[0,1)
$$

The set of hyperarithmetical subsets of $K$ (i.e. the set of images under $\varphi$ of the hyperarithmetical subsets of $[0,1))$ is an effective version of the Borel hierarchy, (see [Hi], [Ma] or [Sh]).

We prove (Theorem 2.1) that "being generic w.r.t. all hyperarithmetical properties in $K$ (resp., in $[0,1)$ )" is not itself a hyperarithmetical property in $K$ (resp., in $[0,1))$.

We hope this is a contribution also to the investigation of the notion of "randomness" (compare with [Lö]); in fact we believe that measure preserving ergodic transformations must have a role in such investigation. 
1. Generic elements in ZFGC. Let $T$ be a measure preserving ergodic transformation of $(G, \mathscr{B}, m)$ where $G$ is an infinite compact abelian group with normalized Haar measure $m$ on the collection $\mathscr{B}$ of Borel sets; also let $\mathcal{L} \subseteq \mathscr{B}$ be a countable field of subsets of $G$, and

$$
G(\mathfrak{L})=\left\{g \in G \mid \forall B \in \mathcal{L}, m(B)=\lim _{n \rightarrow \infty} n^{-1} \sum_{i=0}^{n-1} \chi_{B}\left(T^{i}(g)\right)\right\},
$$

where $\chi_{B}$ denotes the indicator of $B$; in other words, $g \in G(\mathcal{L})$ iff $g$ is generic with respect to $\mathcal{L}$.

THEOREM 1.1. Under the above notation, assume that the following further conditions hold:

(i) (weak choice): $\forall B \in \mathcal{L}$, if $m(B)=1$ then $\exists g \in B$ with $\{g\} \in \mathcal{L}$;

(ii) (T-closure): $\forall g \in G$, if $\{g\} \in \mathcal{L}$ then $\cup_{i \in \omega}\left\{T^{i}(g)\right\} \in \mathcal{L}$.

Then $G(\mathcal{E})$ is Borel and $m(G(\mathcal{E}))=1$, but $G(\mathfrak{L}) \notin \mathcal{L}$.

Proof. The first two conclusions of the theorem immediately follow from Birkhoff's theorem in view of the fact that $|\mathscr{L}|<\omega$. To complete the proof of the theorem, assume that $G(\mathcal{L}) \in \mathcal{L}$ (absurdum hypothesis); by weak choice, $\exists g \in$ $G(\mathcal{L})$ such that $\{g\} \in \mathcal{L}$; by the invariance of $m$ and infinity of $G$, we also have that $m(\{g\})=0$; let $F \subseteq G$ be defined by

$$
F=\bigcup_{i \in \omega}\left\{T^{i}(g)\right\}
$$

Then $F \in \mathcal{L}$ by $T$-closure, and $m(F)=0$. Let $\chi_{F}$ be the indicator of $F$; then

$$
1=n^{-1} \lim _{n \rightarrow \infty} \sum_{i=0}^{n-1} \chi_{F}\left(T^{i}(g)\right) \neq m(F)=0 .
$$

This shows that $g$ is not generic with respect to $F \in \mathcal{L}$, hence, a fortiori, $g \notin G(\mathcal{E})$, a contradiction. Q.E.D.

As a first application of Theorem 1.1, adopting the above stipulations for $(G, \mathscr{B}, m)$, consider the particular case in which $\mathcal{L} \subseteq \mathscr{B}$ is defined by

$$
\mathcal{L}=\{B \in \mathscr{B} \mid B \text { is ZFGC-definable }\} \text {. }
$$

Let $T: G \rightarrow G$ be a measure preserving ergodic transformation satisfying the following condition:

$$
\forall g \in G \text {, if }\{g\} \text { is ZFGC-definable, then so is } \bigcup_{i \in \omega}\left\{T^{i}(g)\right\} .
$$

Then we have the following:

COROLlaRY 1.2. Under the above stipulations, the set $G(\mathfrak{E})$ of those $g \in G$ which are generic w.r.t. $\mathcal{L}$ is a Borel subset of $G$ of measure 1 which is not ZFGC-definable.

Proof. Immediate from Theorem 1.1: $T$-closure is ensured by condition ( + ) above, and weak choice follows from our introductory discussion about ZFGC and the fact that singletons in $G$ are Borel. Q.E.D.

REMARK 1.3. The above corollary may be paraphrased by saying that "being generic w.r.t. ZFGC-definable Borel properties" is a non-ZFGC-definable Borel 
property. The fact that ZFGC has Global Choice rather than just weak choice, yields the following stronger form of Corollary 1.2:

COROLlaRY 1.4. Under the above stipulations, we have that for any $S \subseteq G(\mathcal{E})$ with $S \neq \varnothing, S$ is not ZFGC-definable.

Proof. Use Global Choice for $S$, then argue as in Corollary 1.2. Q.E.D.

REMARK 1.5. The above corollary is paraphrased by saying that there is no nontrivial definable set of ZFGC which is made only of generic elements with respect to the definable Borel properties of ZFGC.

2. An application to effective descriptive set theory. Let $G=K \subseteq \mathrm{C}$ be the unit circle with complex multiplication, natural topology, normalized Haar measure $m$ on the collection $\mathscr{B}$ of Borel sets; let $T: K \rightarrow K$ be given by $T(z)=z^{2}$ for all $z \in K$; let $\lambda: K \rightarrow[0,1)$ isomorphically embed $K$ onto $[0,1)$, with $\lambda=\varphi^{-1}$ and $\varphi$ : $[0,1) \rightarrow K$ given by

$$
\varphi(\theta)=e^{2 \pi i \theta}, \quad \forall \theta \in[0,1) .
$$

Under map $\lambda, m$ becomes Lebesgue measure, multiplication becomes addition $(\bmod 1)$ and $T$ becomes the doubling function $D$ given by $D(\alpha)=2 \alpha(\bmod 1)$ for all $\alpha \in[0,1)$. Notice further that $\lambda$ is measure preserving and $T \circ \varphi=\varphi \circ D .[0,1)$ may be naturally regarded as the set of all dyadic sequences $\alpha: \omega \rightarrow\{0,1\}$ in which zero occurs infinitely often; thus $D(\alpha)$ is obtained from $\alpha$ by cutting down the first digit $\alpha(0)$. Let $\mathcal{L} \subseteq \mathscr{B}$ be the collection of all hyperarithmetical subsets of $K$, i.e. the collection of images under $\varphi$ of all hyperarithmetical subsets of $[0,1)$ (see [Hi], [Ma] or [Sh]). Let $G(\mathcal{L})$ be the set of those $g \in G$ which are generic w.r.t. $\mathcal{L}$.

THEOREM 2.1. Under the above hypotheses, $G(\mathcal{L}) \notin \mathcal{L}$.

Proof. It is well known that $(G, \mathscr{B}, m)$ is an infinite compact Abelian group with $T$ measure preserving and ergodic (see [Wa]); also, $\mathcal{E}$ is a countable field of subsets of $G$ (see [Hi] or [Sh]).

Claim 1 (Weak choice): $B \in \mathcal{L}, m(B)=1$ implies $\exists g \in B$ with $\{g\} \in \mathcal{L}$.

Proof. Every hyperarithmetical set of positive $(>0)$ measure contains a hyperarithmetical singleton, by a result due independently to [Sa] and [Ta] (see also [Ke, p. 368]); now note that $\varphi$ and $\lambda$ are measure preserving.

Claim 2 (T-closure): $\forall g \in G$, if $\{g\} \in \mathcal{L}$, then $\cup_{i \in \omega}\left\{T^{i}(g)\right\} \in \mathcal{L}$.

Proof. Let $g \in G,\{g\} \in \mathcal{L}$ and $\gamma=\lambda(g)$; then $\{\gamma\}$ is a hyperarithmetical singleton, hence a $\Sigma_{1}^{1}$ singleton, say

$$
\alpha \in\{\gamma\} \leftrightarrow \exists \beta \forall b B(\alpha(b), \beta(b), b), \quad \forall \alpha \in[0,1)
$$

with $B$ recursive; (here we follow the usual notation, see [Sh]). Now, let $F=$ $\cup_{i \in \omega}\left\{D^{i}(\gamma)\right\}$ and $\theta \in[0,1)$; then

$$
\begin{aligned}
\theta \in F & \text { iff } \exists n \theta \in\left\{D^{n}(\gamma)\right\} \\
& \text { iff } \exists n \exists \sigma \quad D^{n}(\sigma)=\theta \wedge \sigma \in\{\gamma\} \\
& \text { iff } \exists n \exists \sigma \quad(\sigma \in\{\gamma\} \wedge \forall p \quad \theta(p)=\sigma(n+p))
\end{aligned}
$$


which shows that $F$ is $\Sigma_{1}^{1}$, in view of (1), after some standard manipulations (see [Sh]); on the other hand:

$$
\begin{array}{lll}
\theta \in F & \text { iff } \forall \eta & \left(\eta \in\{\gamma\} \rightarrow \exists n \quad \theta=D^{n}(\eta)\right) \\
& \text { iff } \forall \eta \quad(\eta \notin\{\gamma\} \vee \exists n \forall p \quad(\theta(p)=\eta(p+n)))
\end{array}
$$

which shows that $F$ is $\Pi_{1}^{1}$, in view of (1), after some standard manipulations; so $F$ is $\Delta_{1}^{1}$, hence hyperarithmetical, and so is, by definition, $\varphi(F)=\cdot \cup_{i \in \omega}\left\{T^{i}(g)\right\}$, in view of $T \circ \varphi$ being equal to $\varphi \circ D$.

Having proved Claims 1 and 2 , we see that $\mathcal{L}$ satisfies both weak choice and $T$-closure, so we conclude the proof by applying Theorem 1.1. Q.E.D.

REMARK. The proof of Theorem 2.1 also yields that "being generic w.r.t. all the hyperarithmetical properties of dyadic sequences in $[0,1)$ " is not itself a hyperarithmetical property of dyadic sequences of $[0,1)$. The same holds for arithmetical, instead of hyperarithmetical, properties (see [Ke, p. 370]). The results of this paper can be generalized to probability spaces without using the group structure. The author thanks J. Mycielski for pointing this out to him.

\section{REFERENCES}

[DGS] M. Denker, C. Grillenberger and K. Sigmund, Ergodic theory on compact spaces, Lecture Notes in Math., vol. 527, Springer-Verlag, Berlin and New York, 1976.

[Fe] U. Felgner, Comparison of the axioms of local and universal choice, Fund. Math. 71 (1971), 43-62.

[Fr] N. A. Friedman, Introduction to ergodic theory, Van Nostrand, Amsterdam, 1971.

[Ga] H. Gaifman, Global and local choice functions, Israel J. Math. 22 (1975), 257-265.

[Ha] P. R. Halmos, Measure theory, Springer-Verlag, Berlin and New York, 1974.

[Ke] A. S. Kechris, Measure and category in effective descriptive set theory, Ann. Math. Logic 5 (1973), 337-384.

[Le] A. Levy, Basic set theory, Springer-Verlag, Berlin and New York, 1979.

[Lö] P. Martin Lö, On the notion of randomness, Intuitionism and Proof Theory (Kino, Myhill, Vesley, eds.), North-Holland, Amsterdam, 1970, pp. 73-78.

[Ma] D. A. Martin, Descriptive set theory: projective sets, Handbook of Math. Logic (Barwise, ed.), North-Holland, Amsterdam, 1977, pp. 783-815.

[Po] L. Pontrjagin, Topological groups, Gordon and Breach, New York, 1966.

[Sa] G. E. Sacks, Measure-theoretic uniformity in recursion theory and set theory, Trans. Amer. Math. Soc. 142 (1969), 381-420.

[Sh] J. R. Shoenfield, Mathematical logic, Addison-Wesley, Reading, Mass., 1967.

[Ta] H. Tanaka, A basis result for $\Pi_{1}^{1}$ sets of pasitive measure, Comment. Math. Univ. St. Paul 16 (1968), 115-127.

[Wa] P. Walters, Ergodic theory-introductory lectures, Lecture Notes in Math., Vol. 458, SpringerVerlag, Berlin and New York, 1975.

[Hi] P. G. Hinman, Recursive-theoretic hierarchies, Springer-Verlag, Berlin and New York, 1977.

National Research Council, Loc. Romola N. 76, 50060 Donnini, Florence, Italy 